\title{
Addendum: Finite Size Effects for Some Bootstrap Percolation Models ${ }^{1}$
}

\section{A. C. D. van Enter, ${ }^{2}$ Joan Adler, ${ }^{3}$ and J. A. M. S. Duarte ${ }^{4}$}

Received August 22, 1990

In a recent paper we presented a description of the approach to the infinitesystem limit for some bootstrap percolation (BP) models. Our results for

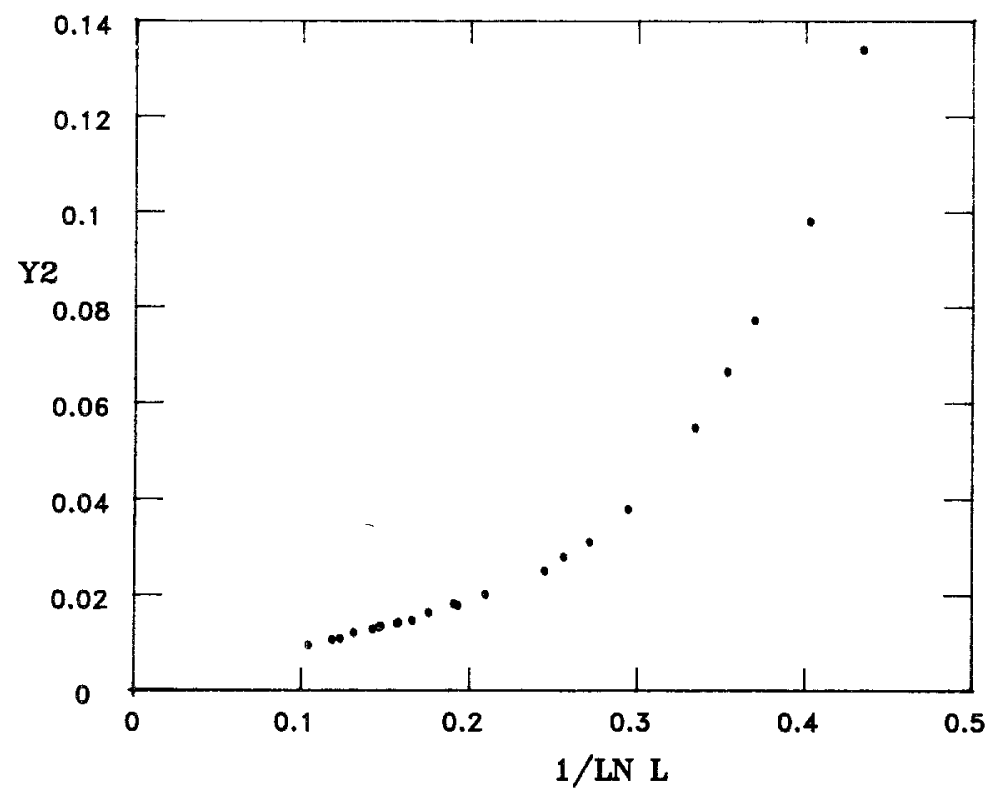

Fig. 1. A graph of $Y 2=\left(q_{50}^{L}\right) /\left[\ln \left(q_{50}^{L}\right)\right]^{2}$ as a function of $1 / \ln L$ for $m=2$ directed BP.

${ }^{1}$ This paper appeared in J. Stat. Phys. 60:323 (1990).

${ }^{2}$ Institute of Theoretical Physics, RUG, P.O. Box 800 , Groningen, The Netherlands.

${ }^{3}$ Department of Physics, Technion, Haifa, 32000, Israel, and School of Physics and Astronomy, Tel Aviv University, Ramat Aviv, 69132, Israel.

${ }^{4}$ CIUP and CFUP F. de Ciencias, 4000 Porto, Portugal. 
one case, the $m=2$ directed BP model, were inconclusive. For this model ${ }^{(1)}$ it was observed in our paper that the numerical data appeared to give a straight line in agreement with scaling of the finite system threshold, $p_{50}^{L}$, toward the infinite-system limit $p_{c}$, according to the rule $1-p_{50}^{L}$ $\sim 1 /(\ln L)^{1 / 2}$, whereas the improved exact result of Schonmann ${ }^{(2)}$ should give $1 / \ln L$ scaling with possible logarithmic corrections. We did not obtain a graph of the behavior with explicit logarithmic corrections, and we were unable to see the approach to the asymptotic $p_{c}=1.0$.

A careful interpretation of Eq. (10) of our paper shows that a possible realization of the logarithmic corrections would give finite-size scaling of the form

$$
\frac{q_{50}^{L}}{\left(\ln q_{50}^{L}\right)^{2}} \sim \frac{1}{\ln L}
$$

where $q=1-p$ and $q_{50}^{L}=1-p_{50}^{L}$. A graph of this behavior is shown in Fig. 1, were we see that the system threshold approaches 1.0 as the system size becomes infinite. The approach is in a straight line for the larger samples. This supports the conjecture that logarithmic corrections are indeed present and makes the numerical results compatible with Schonmann's exact value $p_{c}=1.0$.

\section{ACKNOWLEDGMENTS}

We thank the Israel Academy of Science, the U. S.-Israel Binational Science Foundation, the Technion VPR Fund, the Venezuela Technion Society Fund, and the Royal Netherlands Academy of Arts and Sciences (KNAW) for support. We thank Roberto Schonmann for helpful advice and D. Stauffer for comments on a preliminary version of these results. J. A. acknowledges hospitality at Gröningen and Jülich during the completion of these calculations.

\section{REFERENCES}

1. J. A. M. S. Duarte, Physica A 157:1075 (1989).

2. R. H. Schonmann, private communication. 